\title{
Modification of Transparent Materials by Tightly Focused Annular, Radially and Azimuthally Polarized Ultrafast Laser Beams
}

\author{
Jingyu Zhang, Mindaugas Gecevičius, Martynas Beresna, Peter G. Kazansky \\ Optoelectronics Research Centre, University of Southampton, SO17 1BJ, United Kingdom \\ jz2e11@orc.soton.ac.uk
}

Recently, strong longitudinal electric fields have raised great interest in the laser fabrication, microscopy and optical data storage [1]. Here we demonstrate high quality cylindrically polarized annular beam produced by ultrafast laser written spatially variant polarization converter. We observed that nanogratings cannot be formed by strong longitudinal electric fields, created by a ring-shaped radially polarized beam. In addition, the stronger transverse electric field of azimuthal polarization is demonstrated to produce larger retardance.

The generation of these beams is achieved by an electrically addressed liquid crystal spatial light modulator and a spatially variant polarization converter (S-waveplate), which was fabricated by laser-induced nanostructuring of silica glass [2] (Fig.1 (a)-(c)). The intensity distribution was modelled using the algorithm based on a previous study [3] (Fig.1(d)). Based on simulations, the radially polarized beam should exhibit a longitudinal electric field, which is twice as high as the transverse component with a long depth of focus $(\sim 24 \lambda)$. However, the intensity of the transverse electric field of the annular azimuthally polarized beam is $65 \%$ higher than the radially polarized one.

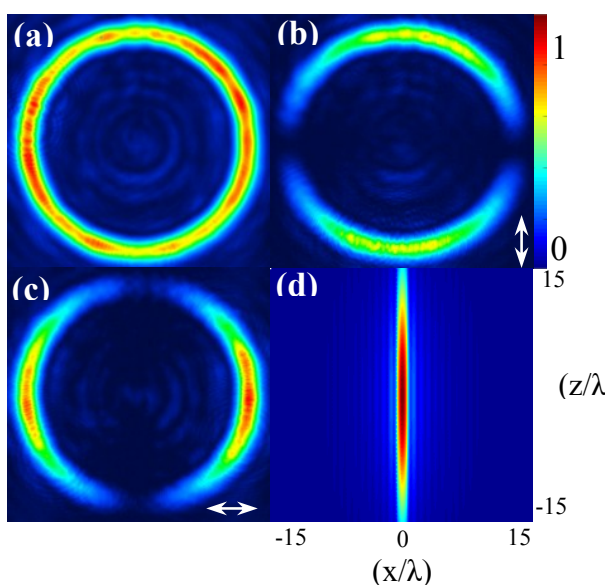

Fig. 1 (a)-(c) are the normalized intensity distributions measured by the CCD camera: (a) the radial ring-shape beam, (b) and (c) is the intensity distributions through linear polarizers. The arrays indicate the transmission axis of the polarizer. (d) Intensity distribution in the xz plane when the radially polarized beam is focused with a $1.2 \mathrm{NA}$ water immersion objective lens. (e) Side view microscope images of bubbles fabricated by a single annular azimuthally and radially polarized laser pulse. (f) and (g) is the birefringence characterization of the structure induced by 10000 laser pulses: (f) pseudo-color indicates orientation of slow axis and (g) represents the retardance value of the structure. (e)-(g) The laser is incident from the top.

Single pulse measurements of the two ring shaped beams were investigated (Fig. 1(e)). The radially polarized beam induced bigger bubbles than the azimuthal beam indicating higher peak intensity of the annular radially polarized beam. However in multiple pulse regime, form birefringence exhibited by laser induced nanogratings was observed for both polarizations and slow axis was aligned perpendicularly to the orientation of the electric field (Fig.1 (f),(g)) [4]. However, no laser-induced nanogratings were formed perpendicular to the laser propagation direction (Fig.1 (f)). Thus, nanogratings could not be induced by the strong longitudinal electric field of the ring-shaped radially polarized beam. A two-lobe structure induced by the ring-shaped radially polarized beam was also observed from the side due to the cylindrical distribution of the nanogratings. Furthermore, the radial beam has a considerably smaller retardance than its counterpart. This is because the longitudinal electric field was not involved in forming the nanogratings and the ring-shaped azimuthally polarized beam has a stronger transverse component than the ring-shaped radially polarized beam.

\section{References}

[1] H. Wang, et al., "Creation of a needle of longitudinally polarized light in vacuum using binary optics." Nature Photon. 2,501-505 (2008)

[2] M. Beresna, et al., "Radially polarized optical vortex converter created by femtosecond laser nanostructuring of glass." Appl. Phys. Lett. 98, 201101 (2011).

[3] K. S. Youngworth and T. G. Brown, "Focusing of high numerical aperture cylindrical vector beams," Opt Express. 7, 77 (2000).

[4] Y. Shimotsuma, et al., "Self-organized nanogratings in glass irradiated by ultrashort light pulses," Phys. Rev. Lett. 91, 247705 (2003). 